\title{
FLOW REDUCTION MARCHING CUBES ALGORITHM
}

\author{
Přemysl Kršek \\ Department of Computer Graphics and Multimedia, \\ Faculty of Information Technology, Brno University of Technology, Czech republic \\ krsek@fit.vutbr.cz
}

\begin{abstract}
The article focuses on modification of traditional Marching cubes algorithm for medical applications. Main disadvantage of the traditional algorithm is size of models. A reduction process has been added to the MC to produce much smaller models. The reduction process is applied during the MC, after processing of each input data layer. Resulting models are 4-5 times smaller in only doubled time.
\end{abstract}

Keywords: marching cubes; CT; MR; discrete data; 3D; human tissue model; reduction

\section{Introduction}

Creation of three-dimensional (3D) geometrical models of human tissues is very useful for medical applications (surgery simulations and planning, implants design, navigations in orthopedic, stomatology, aesthetic surgery, neurosurgery etc.). CT (Computer tomography) and MR (Magnetic resonance) data are main input information about human tissues structure and geometry. It is possible to get tissues voxel models [3] through the data segmentation. The voxel models have discrete data representation [1][2], but the tissues geometry models have vector based representation [1][2]. Therefore, it is necessary to convert discrete voxel models into vector geometry models. A traditional "Marching cubes" (MC) algorithm [6] is generally used for the conversion. It is able to fully automatically produce closed and oriented triangular meshes, without respect to geometry complexity.

For medical applications, it is important to work with resolution on level of input CT/MR data (on voxel size, 0.2-1.0 mm). Consequently, the models have a lot of triangles, typically from hundreds thousand to millions triangles. Consequent operations (smoothing, decimation etc.) with so large models are very heavy on computer memory and CPU power. Therefore, it is useful to find more sophisticated method with same properties as the traditional MC algorithm, but producing models with much lower number of triangles. 


\section{Methods}

\section{Actually used method}

Actually I am using a creation method [3] which consist of several steps:

- Polygonal models generation by the traditional $\mathrm{MC}$ algorithm. Input tissues voxel models are segmented CT/MR data (Fig. 1).

- Smoothing of polygonal models, because MC algorithm produce a little bit layered surfaces with locally flat areas [4][5] (Fig. 1).

- Reduction of smoothed polygonal model by some decimation algorithm [7][8], with level of reduction about 95\% (Fig. 1).

First I have created very fine polygonal models with number of triangles. Next, after smoothing, I have reduced the models with maximal saving of their geometry. Geometry error of reducing process is about $0.05 \mathrm{~mm}$ [8]. Consequently, the error is smaller then size of input voxels and has not influence on required level of detail.
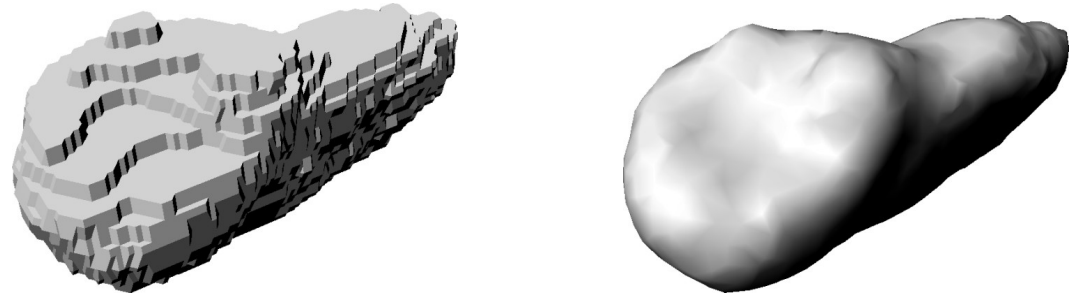

Figure 1. Eye-tooth polygonal model generated by actually used method, after traditional MC (left), smoothed and decimated (right)

\section{Flow reduction $\mathrm{MC}$ algorithm}

The traditional MC algorithm satisfies almost all my needs, except low models size. Therefore, modification of the one will be good solution, as a combination of the traditional MC algorithm and reduction process.

It is not so effective to create many triangles and next reduce them. Therefore main idea of my modified MC algorithm (Flow reduction MC algorithm) is to do $\mathrm{MC}$ process layer by layer (in discrete space) and after each layer make maximal reduction of locally flat areas of previous layer (Fig. 2). The number of triangles created in time of MC process and size of resulted models are consequently much lower then in the case of the traditional MC algorithm. 


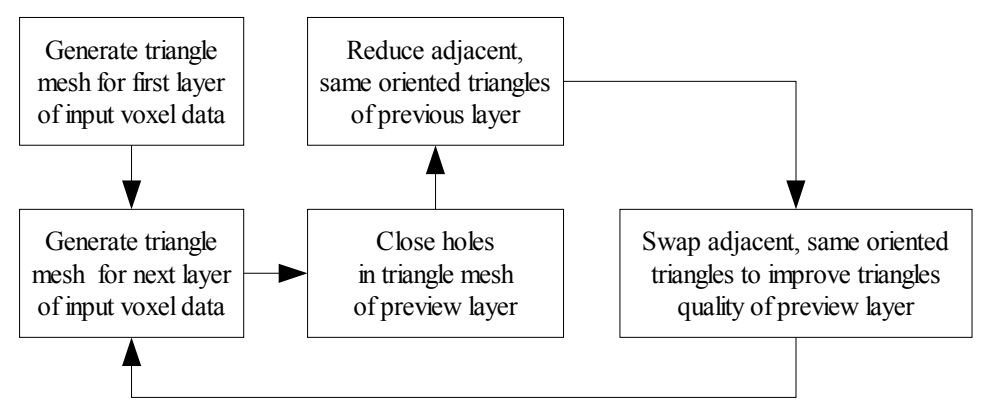

Figure 2. Simplified block diagram of Flow reduction MC algorithm

\section{Reduction process}

For models generated by MC algorithm it is typical, that they have layered surfaces with locally flat areas (Fig. 1). In my "Flow reduction MC algorithm" I maximally reduce triangles in the flat areas. It means that all vertices on the flat areas and on straight edges are removed. Only vertices in corners will stay (Fig. 4). To reduce triangles I have used "Edge collapse" method [8] (Fig. 3), which implicitly save closed and oriented triangular meshes.

The reduction process removes all the redundant triangles in the flat areas with no respect to quality of new triangles [9][10]. Therefore, edge swapping of the triangles with the same orientation has been applied to improve their quality [11][12] (Fig. 2, 4).

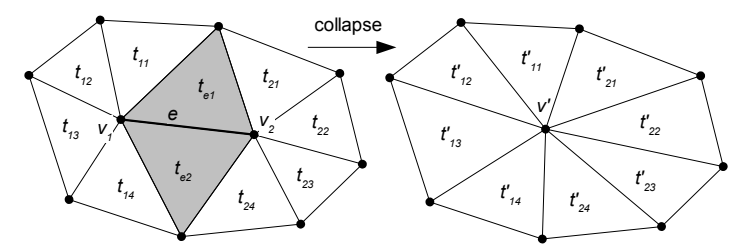

Figure 3. Scheme of edge collapse method
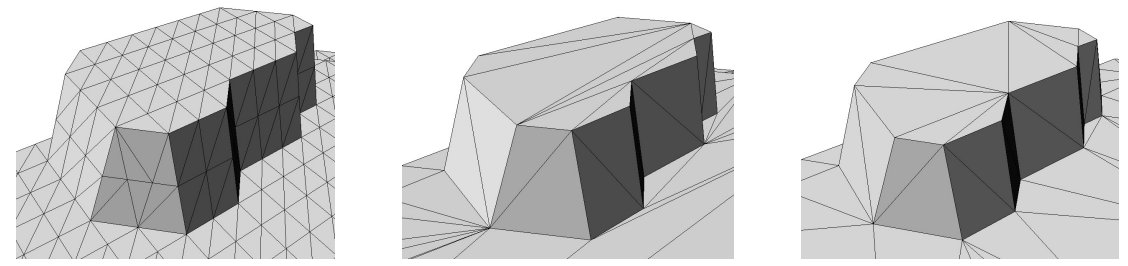

Figure 4. Tooth model detail: produced by traditional MC algorithm (left), produced by Flow reduction $\mathrm{MC}$ algorithm with out edge swapping (middle) and after edge swapping (right) 


\section{Results}

All MC algorithms were implemented in $\mathrm{C}++$ and compiled by $\mathrm{C} / \mathrm{C}++$ compiler MinGW 3.3.1. All the presented tests were run on a $\mathrm{PC}$ with $2.4 \mathrm{GHz}$ P4 CPU, 1024 MB RAM, 120 GB HD, Win XP. A group of tissue models from real medical applications was selected as testing objects. Results for five of them (Fig. 5-9) are in Table 1. The table compares number of triangles and creation time for the traditional MC algorithm with Flow reduction MC algorithm. The table also contains triangle numbers and creation time ratios.

Table 1. Result models parameters

\begin{tabular}{||l||c|c|c|c|c|c||}
\hline \multirow{2}{*}{ Model } & \multicolumn{2}{|c|}{ Traditional MC } & \multicolumn{2}{c||}{ Flow reduction MC } & \multicolumn{2}{c||}{ Ratio (Flow / Clasic) } \\
\cline { 2 - 7 } & Triangles & Time [s] & Triangles & Time [s] & Triangles & Time \\
\hline Face & 1049624 & 13 & 207998 & 30 & 0.2 & 2.3 \\
\hline Skull & 957356 & 11 & 263972 & 20 & 0.28 & 1.8 \\
\hline Maxilla & 2637106 & 28 & 658560 & 83 & 0.25 & 3.0 \\
\hline Pelvis & 607996 & 8 & 133036 & 15 & 0.22 & 1.9 \\
\hline Spine & 296372 & 4 & 79620 & 8 & 0.27 & 2.0 \\
\hline
\end{tabular}

\section{Conclusion}

Flow reduction MC algorithm produces from 4 to 5 times smaller polygonal models. The creation process takes 2 to 3 times longer time comparing to the traditional MC. However, the additional processing time is compensated by time savings in the consequently performed operations because much smaller models are processed. The models created by Flow reduction MC represent exactly the same geometry as in the case of the traditional MC algorithm.

Flow reduction $\mathrm{MC}$ algorithm produces the same type of models as the traditional MC - polygonal triangular meshes. Therefore, it is possible to use the models in the consequent operations (smoothing, reducing etc.) and in all applications. Because the models are smaller, the consequent steps are faster and save a lot of memory. Therefore, it is possible to produce bigger or more complex models on the same computer equipment.

Main future plan is to add smoothing process to Flow reduction MC algorithm. Final goal is to develop algorithm which will have the same basic properties as the traditional MC algorithm, but will directly produce prereduced and presmoothed models. 


\section{Acknowledgments}

The work is supported by grant project GP 201/01/D141.

\section{References}

[1] Foley, J., D., et al., Computer Graphics: Principles and Practise, Addison-Wesley, 1992

[2] Watt, A., 3D Computer Graphics, Addison-Wesley, 1993

[3] Nagy. I. Krsek P. Husták. J.: Voxel Model Creation of Human Tissues from CT and MRI Data for Biomechanical Applications. In: Proceedings, Euroconference Biosignal 2000, Brno, FE VUT Brno, 2000, s. 278-280, ISBN 80-214-1610-6

[4] Balendran B., A Direct Smoothing Method For Surface Meshes: Proceedings, 8th International Meshing Roundtable, South Lake Tahoe, USA, 1999, 189-193

[5] Taubin G., Geometric signal processing on polygonal meshes: Eurographics 2000 State of The Art Report(STAR), September 2000.

[6] Lorensen W., Cline H. Marching cubes, A high resolution 3D surface construction algorithm. In Siggraph'87 Conference Proceedings, pages 163-169, 1987

[7] Schroeder W. J., Zarge J. A., Lorensen W. E. Decimation of triangle meshes. In Siggraph'92 Conference Proceedings, pages 65-70, July 1992.

[8] Garland M., Heckbert, P. Surface simplification using quadric error metrics. In Siggraph'97 Conference Proceedings, pages 209-216, 1997.

[9] Berzins M.: Based Mesh Quality for Triangular and Tetrahedral Meshes. In: Proceedings, 6th International Meshing Roundtable, Sandia National Lab, USA, 1997, s. 427-436

[10] Berzins M.: Mesh Quality - A Function of Geometry, Error Estimates or Both. In: Proceedings, 7th International Meshing Roundtable, Sandia National Lab, USA, 1998, s. 229-238

[11] Rivara M. C.: New Mathematical Tools and Techniques for the Refinementand or Improvement of Unstructured Triangulations. In: Proceedings, 5th International Meshing Roundtable, Sandia National Lab, USA, 1996, s. 77-86

[12] Freitag L. A., Gooch C. O.: A Comparison of Tetrahedral Mesh Improvement Techniques. In: Proceedings, 5th International Meshing Roundtable, Sandia National Lab, USA, 1996, s. 87-106
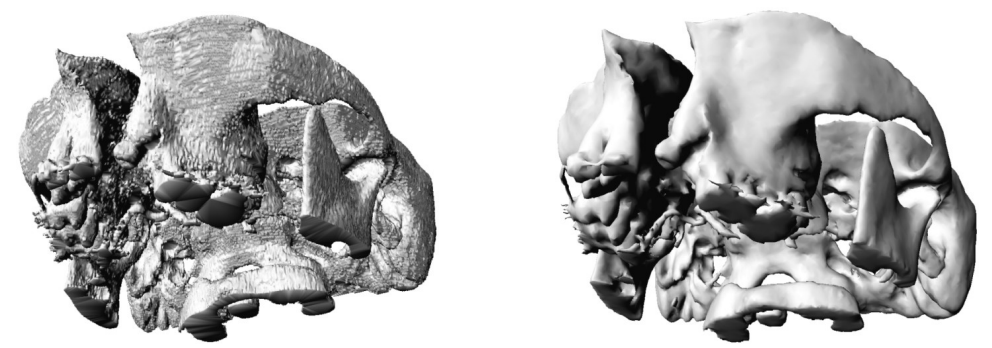

Figure 5. Maxilla Flow MC model (left), smoothed and reduced model (right) 

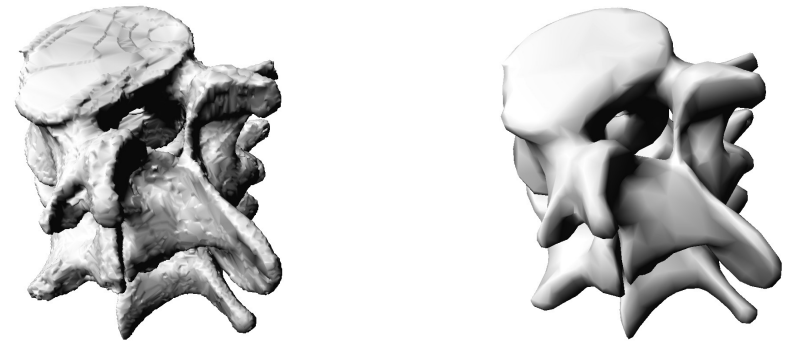

Figure 6. Spine Flow MC model (left), smoothed and reduced model (right)
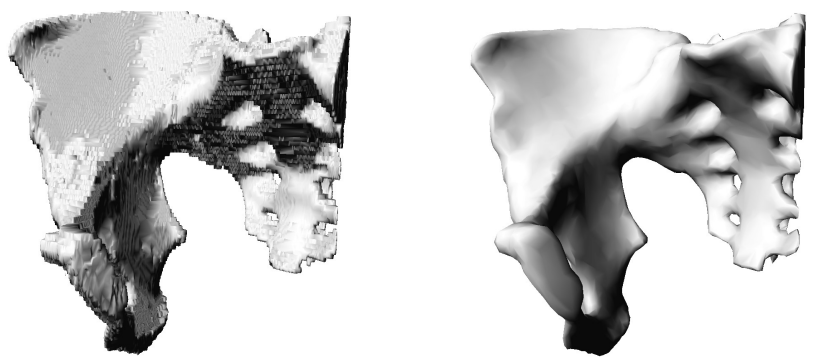

Figure 7. Pelvis Flow MC model (left), smoothed and reduced model (right)
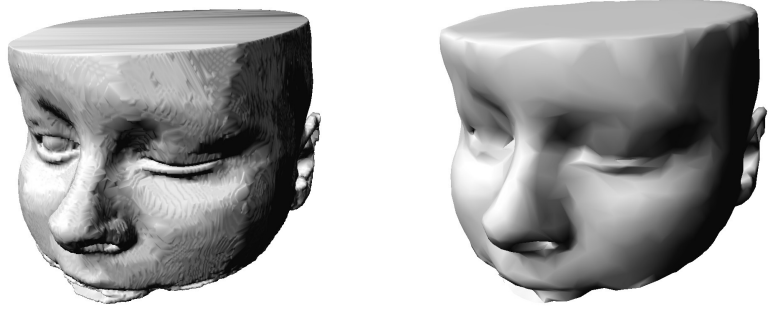

Figure 8. Face Flow MC model (left), smoothed and reduced model (right)
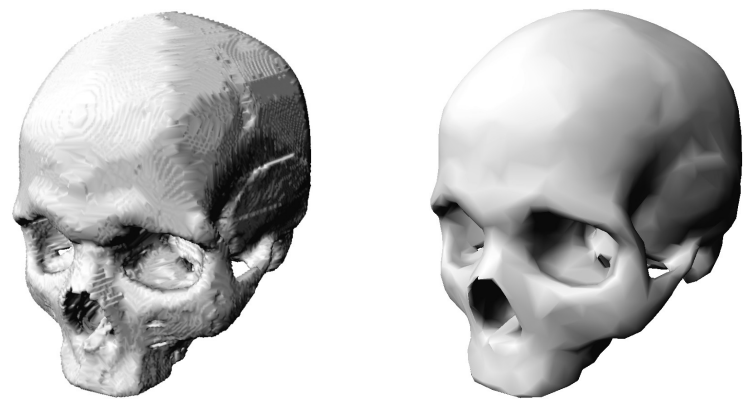

Figure 9. Skull Flow MC model (left), smoothed and reduced model (right) 